\title{
Production of paclitaxel by Fusarium solani isolated from Taxus celebica
}

\author{
B V S K Chakravarthi ${ }^{l}$, Prasanta Das $^{2}, \mathrm{Kalpana} \mathrm{Surendranath}^{l}$, Anjali A Karande ${ }^{l}$ and \\ CHELLIAH JAYABASKARAN ${ }^{l, *}$ \\ ${ }^{1}$ Department of Biochemistry and ${ }^{2}$ Department of Inorganic and Physical Chemistry, Indian Institute of Science, \\ Bangalore 560 012, India \\ *Corresponding author (Fax, 91-80-23600814; Email, cjb@biochem.iisc.ernet.in)
}

\begin{abstract}
A fungus was isolated from the stem cuttings of Taxus celebica, which produced paclitaxel in liquid-grown cultures. The fungus was identified as Fusarium solani based on colony characteristics, morphology of conidia and the 26S rDNA sequence. Paclitaxel was identified by chromatographic and spectroscopic comparison with authentic paclitaxel and its cytotoxic activity towards Jurkat cells in vitro.
\end{abstract}

[Chakravarthi B V S K, Das P, Surendranath K, Karande A A and Jayabaskaran C 2008 Production of paclitaxel by Fusarium solani isolated from Taxus celebica; J. Biosci. 33 259-267]

\section{Introduction}

Paclitaxel, a diterpene compound, has been used alone or in combination with other chemotherapeutic agents for the treatment of a variety of cancers, as well as AIDS-related Kaposi sarcoma (Croom 1995; Brown 2003). It was first isolated from the bark of the Pacific yew tree, Taxus brevifolia (Wani et al 1971), but obtaining paclitaxel from this source requires destruction of trees. The limited supply of the drug has prompted efforts to find alternative sources of paclitaxel. The first report on the isolation of paclitaxelproducing fungi from Taxus brevifolia appeared in 1993 (Stierle et al 1993; Strobel et al 1993). Alternative methods for paclitaxel production, such as chemical synthesis, tissue and cell cultures of the Taxus species are expensive and give low yields (Guenard et al 1993; Frense 2007). Cost-effective paclitaxel production is possible by industrial fermentation. Several paclitaxel-producing fungi have been identified, such as Taxomyces andrenae, Pestalotiopsis microspora, Alternaria sp., Fusarium lateritium and Periconia sp. (Strobel et al 1996; Li et al 1996; Wang et al 2000; Zhou 2001; Guo et al 2006; Xu et al 2006; Yuan et al 2006). Among these,
P. microspora isolated from Taxus wallachiana produces 60-70 $\mu \mathrm{g} / 1$ culture whereas the yield from other species is less than $2 \mu \mathrm{g} / 1$. Recently, Fusarium mairie isolated from China mairie yew yielded $20 \mu \mathrm{g} / 1$ of paclitaxel. This report describes a fungus Fusarium solani isolated from Taxus celebica, which yields $1.6 \mu \mathrm{g} / \mathrm{l}$ of paclitaxel and its precursors baccatin III and 10-deacetylbaccatin III.

\section{Materials and methods}

\subsection{Isolation of $\mathrm{F}$. solani from $\mathrm{T}$. celebica}

Fresh stem cuttings of T. celebica obtained from Bedgeburg, National Pinetum, UK, were defoliated, cut into $2-3 \mathrm{~cm}$ nodal segments, surface sterilized by rinsing with $0.1 \%$ (w/v) $\mathrm{HgCl}_{2}$ for $10 \mathrm{~min}$, followed by 5-6 washings with sterile distilled water. The segments were placed in an upright position on MS inorganic salts (Murashige and Skoog 1962) supplemented with vitamins $+100 \mathrm{mg} \mathrm{l}^{-1}$ myoinositol, $1 \mathrm{mg} \mathrm{l}^{-1}$ benzyl adenine, $3 \%$ sucrose and solidified with $0.8 \%$ agar in $12 \times 5.5 \mathrm{~cm}$ glass bottles (2-3 nodal cuttings per bottle).

Keywords. Apoptosis; endophytic fungus; Fusarium solani; paclitaxel

Abbreviations used: HPLC, high-performance liquid chromatography; ITS, internal transcribed spacer; LC-ESI-MS, liquid chromatography electrospray ionization mass spectra; MALDI, matrix-assisted laser desorption/ionization; PBS, phosphate buffered saline; PCA, potato carrot agar; PCR, polymerase chain reaction; PDA, potato dextrose agar; Rf, Relative front; TLC, thin-layer chromatography 
The cultures were incubated at $25 \pm 2^{\circ} \mathrm{C}$ in darkness. After several days, fungal mycelia emerged from the cut surface of the segments. The mycelium was transferred to water agar. After 7 days, individual hyphal tips were cut and transferred on either potato dextrose agar (PDA) or MID agar medium (Pinkerton and Strobel 1976). The fungal culture was stored in distilled $\mathrm{H}_{2} \mathrm{O}$ at $4^{\circ} \mathrm{C}$ or in $15 \%(\mathrm{v} / \mathrm{v})$ glycerol at $-70^{\circ} \mathrm{C}$ as a suspension of spores and mycelium. The isolated fungus was grown in potato carrot agar (PCA) medium for 10 days. The fungal colonies on PCA were white to off-white, floccose, with irregular margins. The reverse side of the culture was cream in colour. The morphology was examined microscopically following staining with cotton blue in lactophenol (figure 1).

\subsection{Identification of the fungus}

Genomic DNA was isolated from the mycelial mass using methods described elsewhere (Rogers and Bendich 1994).
The internal transcribed spacer (ITS) and D1/D2 26S rDNA regions $(\sim 1.2 \mathrm{~kb})$ were amplified with pITS1 and pNL4 primers. The amplicons were purified using the Qiagen gel extraction kit. Direct sequencing of the gel-purified polymerase chain reaction (PCR) product was performed with primer NL4 using an ABI Big Dye Terminator cycle sequencing ready reaction kit (Applied Biosystems, USA) (figure 2). Processing of the samples for loading onto an ABI Prism $3130 x l$ genetic analyser (Applied Biosystems, USA) sequencer was performed according to the manufacturer's instructions. A sequence similarity search was done using Genbank BLASTN (Altschul et al 1997).

\subsection{Culturing and production of paclitaxel}

The fungus was grown in $500 \mathrm{ml}$ of potato dextrose liquid medium in 21 Erlenmeyer flasks at $25^{\circ} \mathrm{C}$ in the dark in stationary cultures. After 21 days, 11 of culture (medium
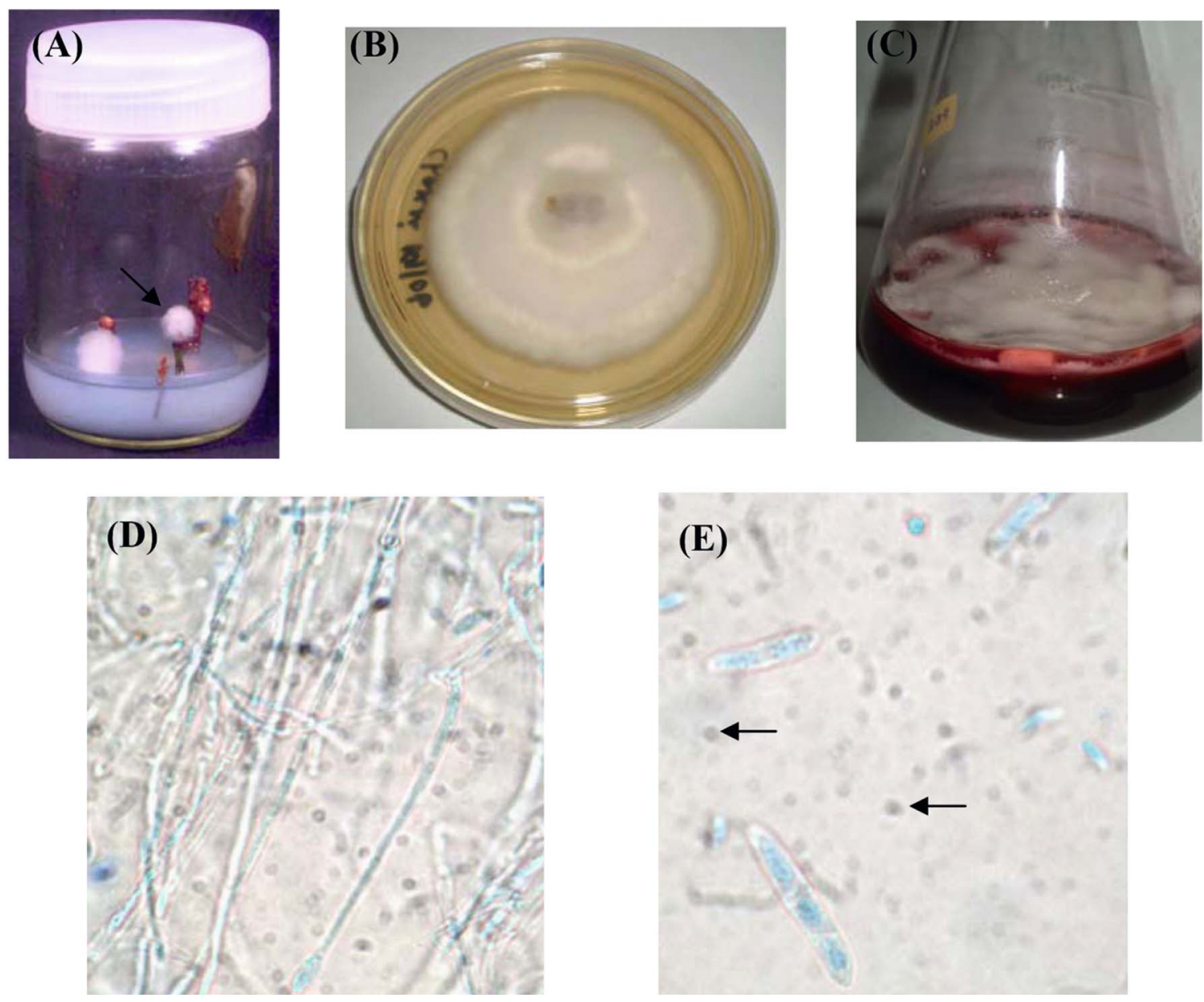

Figure 1. Culture characteristics of F. solani. (A) Mycelium emerging from surface-sterilized stem segments of Taxus cultured in Murashige and Skoog medium. (B) A culture growing on the surface of potato dextrose agar. (C) A 21-day-old stationary culture growing in liquid potato dextrose medium. (D) Photomicrograph of conidiating mycelium. (E) One- to 3-celled macroconidia, and unicellular microconidia (arrow). 
5' -CACCCGGTGTATTCGCAGCATCCTTGCGATGCGCGACCTCAGTCCGCCAC AGGGTATTACGCAACGG GCTATAACACTCCCCGGAGAGAGCCACGTTCCC GATGCCTTTATCCCCCAGGGCGAACTGATGCTGGCCTGAGCCGGAAGAGTG CACCGGGGAGAACCCCGGATGATCAACCAAGCCCAAGTCTGGTCACAAGCG CTTCCCTTTCAACAATTTCACGTACTGTTTAACTCTCTTTTCAAAGTTCTTTTC ATCTTTCGATCACTCTACTTGTGCGCTATCGGTCTCTGGCCGGTATTTAGCTTT AGAAGACATATACCTCCCATTTAGAGCAGCATTCCCAAACTACTCGACTCGT CGAAGGAGCTTTACAGAGGTTCGGCGTCCAACCAGACGGGGCTCTCACCCTC TATGGCGTCCCGTTCCAGGGAACTCGGAAGGCACCTCACCAAAAGCATCCTC TACAAATTACAACTCGGGCCCGAGAGCCAGATTTCAAATTTGAGCTGTTGCC GCTTCACTCGCCGTTACTGGGGCAATCCCTGTTGGTTTCTTTTCCTCCGCTTAT TGATATGCTTAAGTTCAGCGGGTATTCCTACCTGATTCGAGGTCAACTTCAGA AGAGTTGGGTGTTTTACGGCGTGGCCGCGCCGCTCTCCAGTCGCGAGGTGTTA GCTACTACGCGATGGAAGCTGCGGCGGGACCGCCACTGTATTTGGGGGGACG GCGTGTGCCCACGGGGGGCTCCGCCGATCCCCAACGCCAGGCCCGGGGGCCT GAGGGTTGTAATGACGCTCGAACAGGCATGCCCGCCAGAATACTGGCGGGCG CAATGTGCGTTCAAAGATTCGATGATTCACTGAATTCTGCAATTCACATTACT TATCGCATTTCGCTGCGTTCTTCATCGATGCCAGAGCCAAGAGATCCGTTGTT GAAGTTTTAATTTAATTTGCTTTGTTTACTCAGAAGATACACTAAAAGAAACC AGAGTTAATGGTGCGCCTGGCGGGGGCGCCGTTTGTACTGCGACGTCTATTCC ATGCCGACCACGTAAGGTAAGTTCACGTGCATTGCATGTGCTGATACACGTA TGATACTTCACCTGCACTTCGCATCGGAATCGGCAAAGACGGTGAGGCTACC GTAGT- $3^{\prime}$

Figure 2. F. solani internal transcribed spacer regions (ITS) of rRNA gene cluster, partial sequence and D1/D2 domain of 26S rDNA partial sequence.

plus mycelium) was extracted twice with an equal volume of methylene chloride (Strobel et al 1996). The organic phase was taken to dryness under reduced pressure at $35^{\circ} \mathrm{C}$. The residue was dissolved in $1 \mathrm{ml}$ methanol.

\subsection{Thin-layer chromatography (TLC)}

TLC analyses were carried out on Merck $0.25 \mathrm{~mm}$ silica gel plates developed in solvents: solvent A, chloroform: methanol $(7: 1, \mathrm{v} / \mathrm{v})$; solvent $\mathrm{B}$, chloroform:acetonitrile, $(7: 3, \mathrm{v} / \mathrm{v})$; solvent $\mathrm{C}$, ethylacetate:isopropanol $(95: 5, \mathrm{v} / \mathrm{v})$; solvent D, chloroform:acetonitrile $(4: 1, \mathrm{v} / \mathrm{v})$ and solvent E, chloroform:methanol $(9.2: 0.8, \mathrm{v} / \mathrm{v})$. TLC analysis of the methanolic extract of $F$. solani as well as the methanolic solution of paclitaxel standard was carried out using solvent $\mathrm{E}$ by spotting on the start line of a $0.25 \mathrm{~mm}(10 \times$ $20 \mathrm{~cm}$ ) silica gel plate. Paclitaxel was detected using a spray reagent consisting of $1 \%$ vanillin $(\mathrm{w} / \mathrm{v})$ in sulphuric acid after gentle heating (Cardellina 1991). It appeared as a bluish spot which turned dark grey after $24 \mathrm{~h}$. The paclitaxel spot was identified by comigration with standard paclitaxel.

\subsection{High-performance liquid chromatography (HPLC)}

HPLC separation was performed using a Kromasil C18-column $(250 \times 4.6 \mathrm{~mm})$ at a flow rate of $1 \mathrm{ml} / \mathrm{min}$. A $20 \mu 1$ amount of sample was injected. The mobile phase was methanol:water $(70: 30, \mathrm{v} / \mathrm{v})$. The effluent was monitored at $227 \mathrm{~nm}$ (figure 3). 

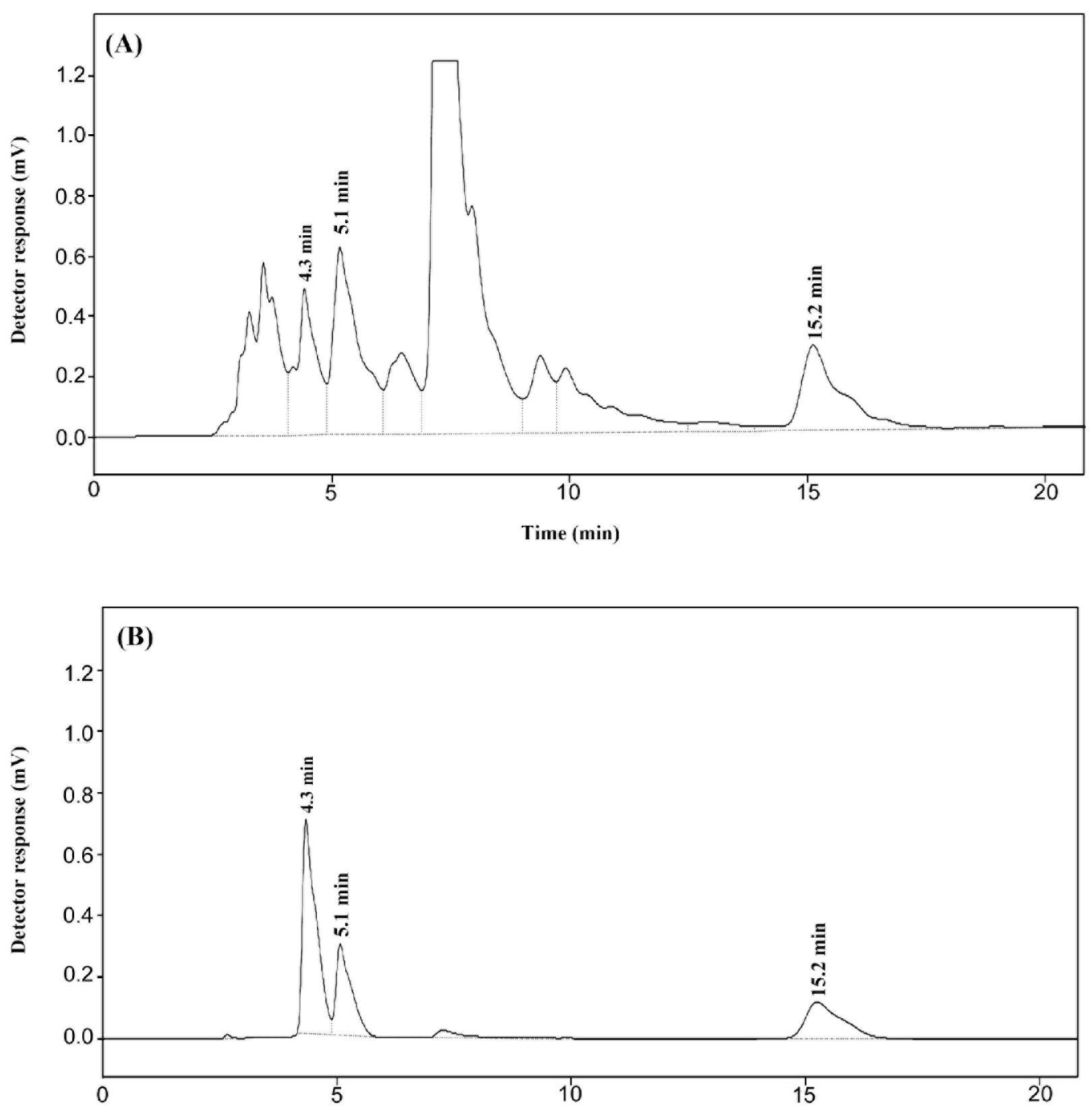

Time (min)

Figure 3. High performance liquid chromatography (HPLC) analysis of the fungal extract (A) and of authentic 10-deacetyl baccatin-III (retention time $=4.3 \pm 0.1 \mathrm{~min})$, baccatin-III (retention time $=5.1 \pm 0.1 \mathrm{~min})$ and paclitaxel standards (retention time $=15.2 \pm 0.1 \mathrm{~min})(\mathbf{B})$. Analysis was performed on a Kromasil $100 \mathrm{C} 185 \mu \mathrm{m}$ column $(4.6 \times 250 \mathrm{~mm})$ using a mobile phase of methanol:water (7:3) containing $0.1 \%$ trifluoroacetic acid (TFA) at a flow rate of $1 \mathrm{ml} / \mathrm{min}$ and absorbance was monitored at $227 \mathrm{~nm}$.

\subsection{Spectroscopic analyses}

After chromatography, the area with silica gel on the plates containing putative paclitaxel was scraped at the appropriate Relative front (Rf) and exhaustively eluted with methanol (figure 4). The sample was analysed by matrix-assisted laser desorption/ionization (MALDI) mass spectroscopy. UV-spectrometry was done on the HPLC paclitaxel fraction between $200 \mathrm{~nm}$ and $500 \mathrm{~nm}$ on a Perkin Elmer (Lambda 35) UV/VIS spectrophotometer (figure 5). Liquid chromato- 
graphy electrospray ionization mass spectra (LC-ESI-MS) were recorded on a Bruker Daltonics Esquire 3000 Plus Ion Trap mass spectrometer attached to an Agilent 1000 series HPLC system (figure 6). The samples were infused into the mass spectrometer through a reverse-phase $\mathrm{C} 18$ column (UltraPac, $4.0 \times 250 \mathrm{~mm}, \mathrm{LKB}$, Sweden) by the application of a gradient

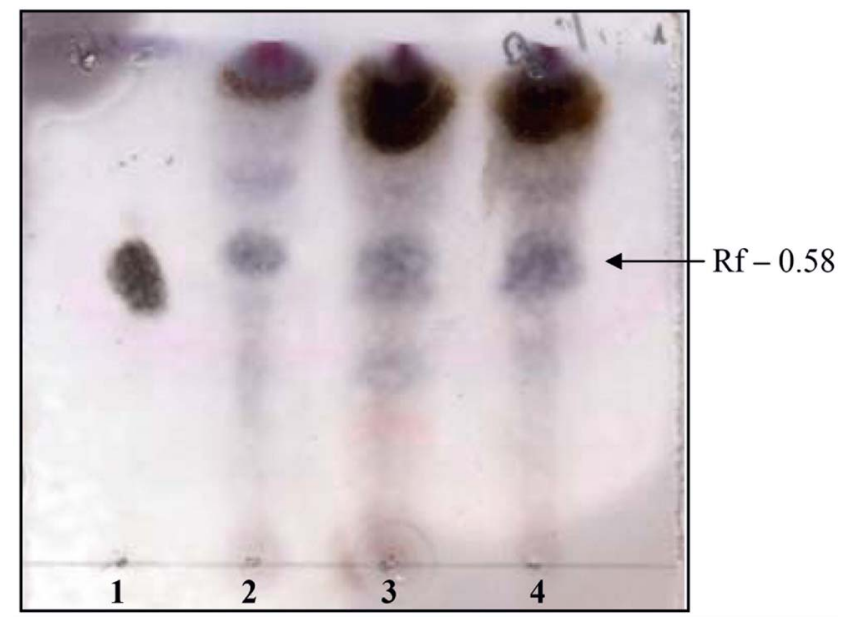

Figure 4. Thin-layer chromatography (TLC) analysis of fungal paclitaxel. Samples were subjected to TLC on a silica (silica $60 \mathrm{~F}_{254}$ ) plate. The chromatogram was developed using the solvents $\mathrm{CHCl}_{3}$ : $\mathrm{CH}_{3} \mathrm{OH}(9.2: 0.8, \mathrm{v} / \mathrm{v})$ and sprayed with $1 \%$ vanillin-sulphuric acid to detect paclitaxel. Lane 1: methanolic solution of paclitaxel standard; lanes 2, 3 and 4 are methanolic extracts of $F$. solani. elution using a binary solvent delivery system (solvent A $-0.1 \%$ formic acid in water and solvent $\mathrm{B}-0.1 \%$ formic acid in acetonitrile) at a flow rate of $0.3 \mathrm{ml} / \mathrm{min}$. The data were acquired over an $\mathrm{m} / \mathrm{z}$ range of $500-1000$ in positive ion mode and analysed using the Esquire analysis software (figure 7).

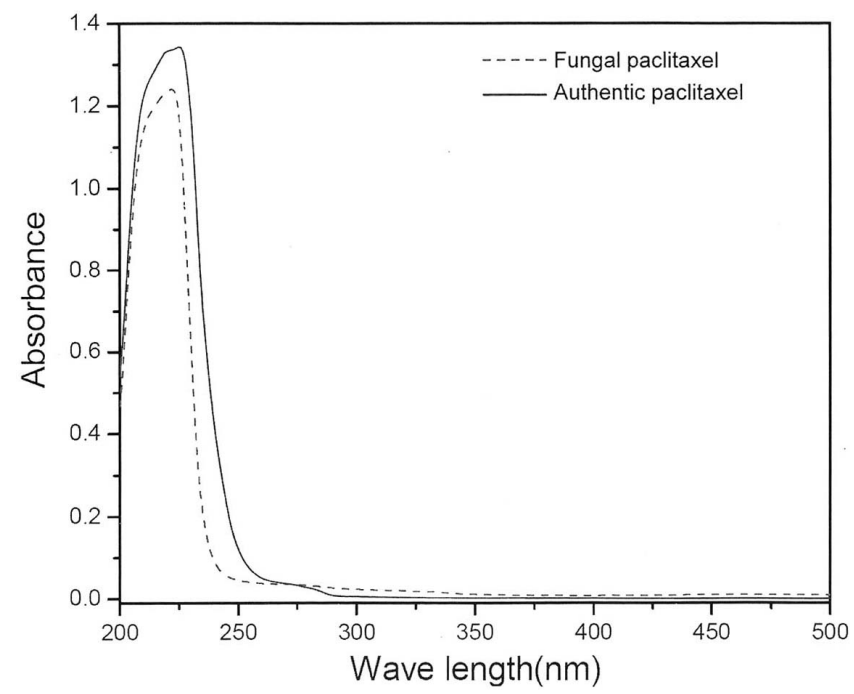

Figure 5. UV absorption spectrum of the peak of the high performance liquid chromatography (HPLC) corresponding to $15.2 \mathrm{~min}$ in figure 3A. Dotted line, fungal paclitaxel; solid line, standard paclitaxel.

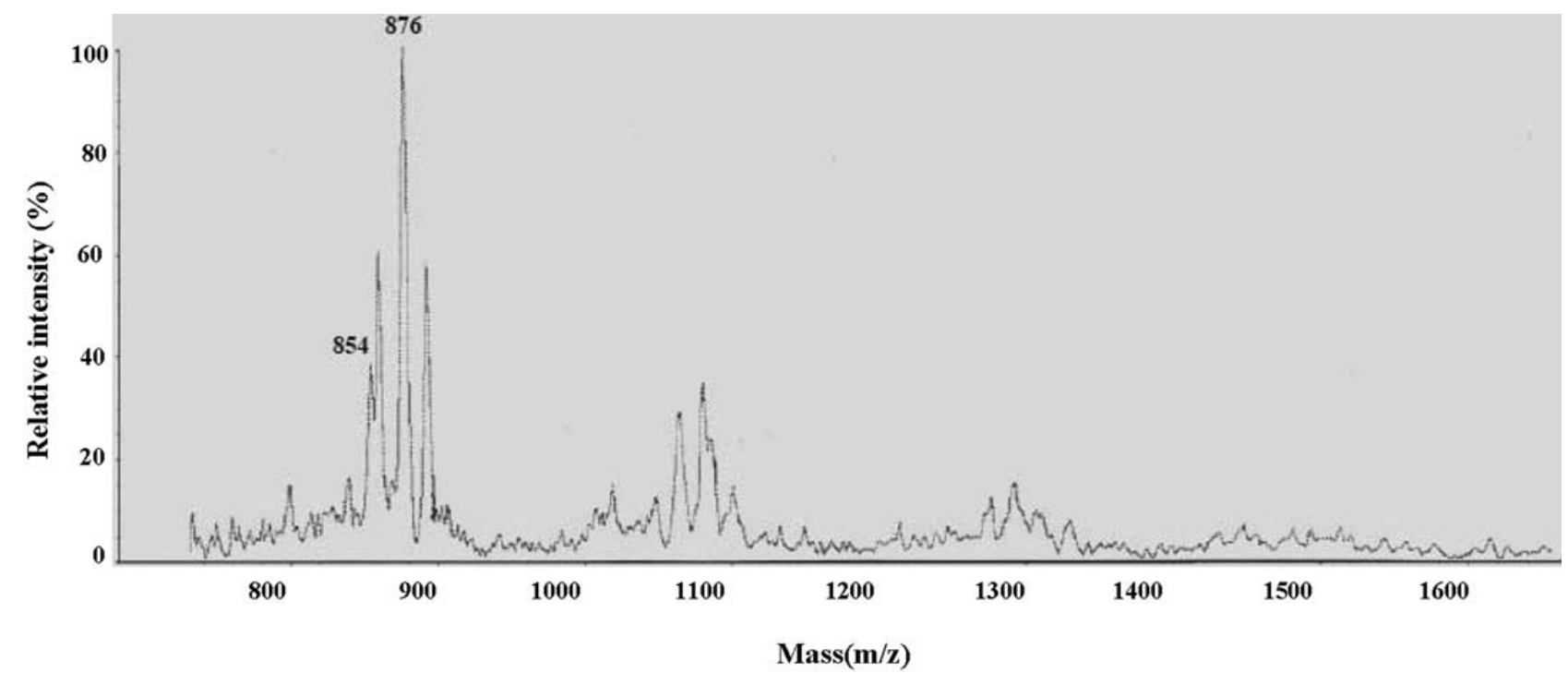

Figure 6. Matrix-assisted laser desorption/ionization mass spectra (MALDI-MS) of thin-layer chromatography (TLC) purified fungal paclitaxel. Note the characteristic peaks in the spectrum at the molecular ion plus $\mathrm{H}^{+}=854$ and the molecular ion plus $\mathrm{Na}^{+}=876$. 


\subsection{Apoptosis assay and FACS analysis}

As described elsewhere (Fraker et al 1994), Jurkat cells $\left(1 \times 10^{6}\right.$ cells $\left./ \mathrm{ml}\right)$ were treated with either $10 \mu \mathrm{M}$ fungal compound or $10 \mu \mathrm{M}$ standard paclitaxel in RPMI-1640 medium supplemented with $10 \%(\mathrm{v} / \mathrm{v})$ foetal bovine serum (FBS) for $24 \mathrm{~h}$. The cells were centrifuged at $300 \mathrm{~g}$ for $5 \mathrm{~min}$, resuspended in $100 \mu \mathrm{l}$ of $50 \mathrm{mM}$ phosphate buffered saline (PBS) and fixed with ice-cold 70\% (v/v) ethanol for $30 \mathrm{~min}$ at $-20^{\circ} \mathrm{C}$. The cells were pelleted and washed once with PBS and stained with ethidium bromide $(50 \mu \mathrm{g} / \mathrm{ml})$ staining solution containing $100 \mu \mathrm{g} / \mathrm{ml}$ RNase A and $1 \%$ (v/v) Triton
$\mathrm{X}-100$ for $60 \mathrm{~min}$ at $37^{\circ} \mathrm{C}$. The cells were then analysed by a fluorescence-activated flow cytometer (FACScan, Becton Dickinson, USA) (figure 8).

\section{Results and discussion}

\subsection{Identity of fungus isolated from $\mathrm{T}$. celebica stem segment}

As shown in figure 1, a white mycelium proliferated into the air from the cut surface of the surface-sterilized
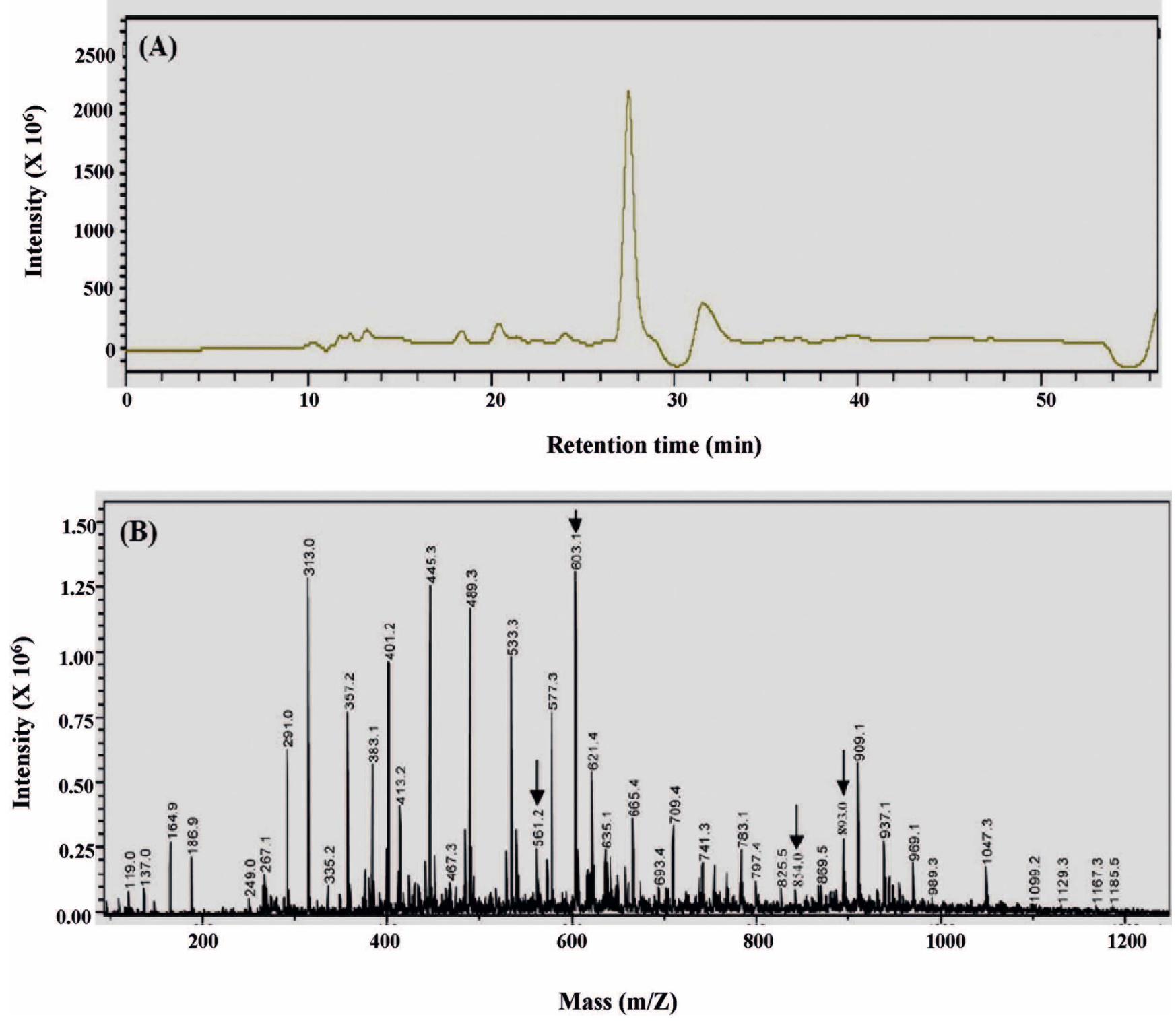

Figure 7. Liquid chromatography electrospray ionization mass spectra (LC-ESI-MS) analysis of crude methylene chloride extract of F. solani. (A) HPLC separation of the compounds from the Fusarium solani culture extract. (B) ESI-MS spectrum obtained in the full scan mode recorded at a cone voltage of $60 \mathrm{~V}$, showing 10-deacetylbaccatin III $\left(\mathrm{M}+\mathrm{NH}_{4}^{+}=561\right)$, baccatin III $\left(\mathrm{M}+\mathrm{NH}{ }_{4}^{+}=603\right)$ and paclitaxel $\left[\left(\mathrm{M}+\mathrm{H}^{+}=854 \& \mathrm{M}+\mathrm{K}^{+}=893\right)\right]$ (arrows). 

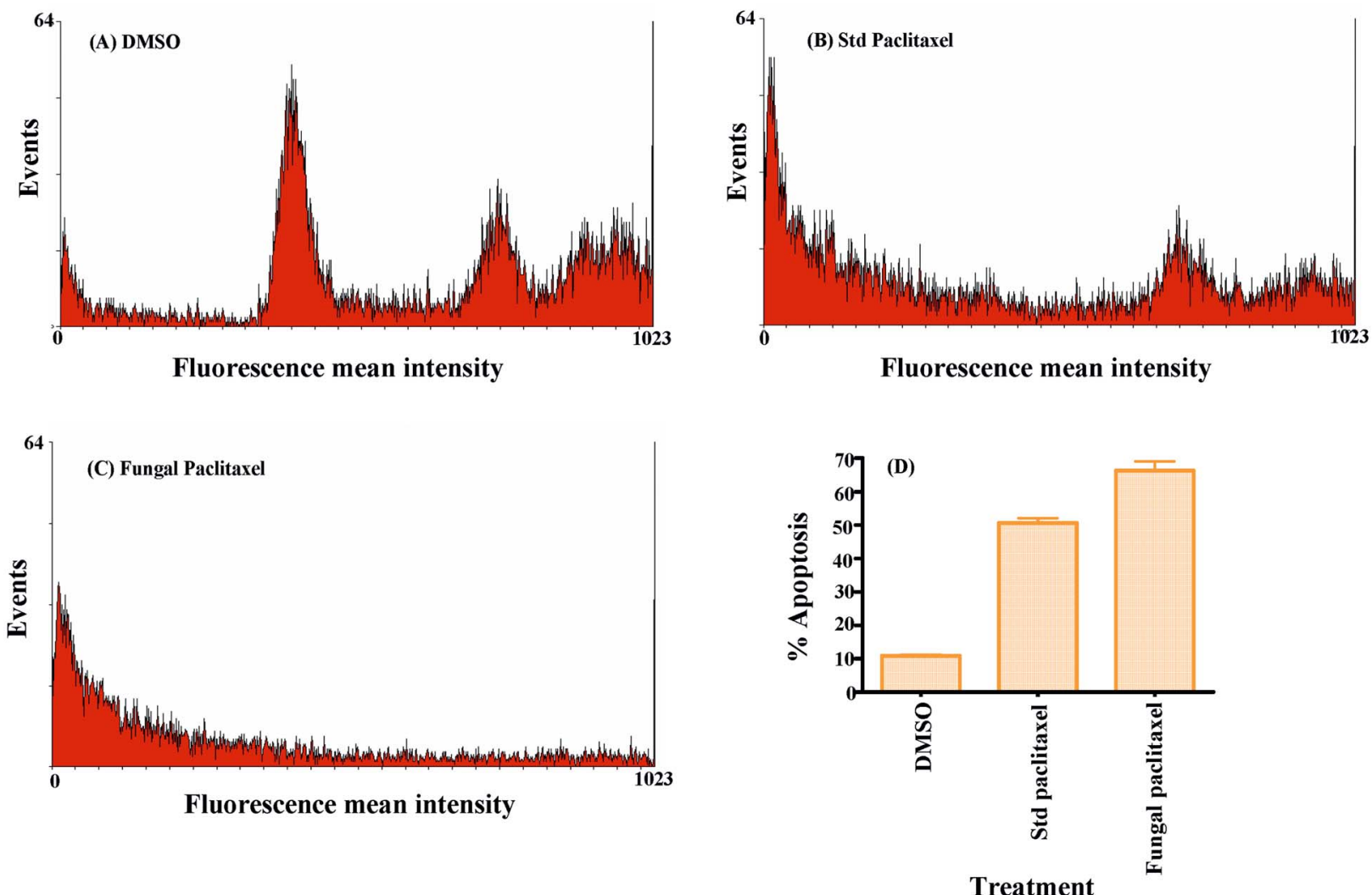

Figure 8. Apoptotic activity of fungal paclitaxel through FACScan analysis. Jurkat cells were cultured with $10 \mu \mathrm{M}$ of the fungal paclitaxel for $24 \mathrm{~h}$. The cells were then fixed in ethanol, stained with ethidium bromide and the percentage of apoptotic population was determined by FACS analysis. (A), (B) and (C): FACS profile; (D) Histogram of the FACS profile. Standard paclitaxel (10 $\mu$ M) was taken as the positive control and only DMSO as the vehicle control.

stem segment. Since the mycelium growth emerged from surface-sterilized stem segments, this growth was assumed to be that of an endophytic fungus. We did not confirm the endophytic nature of the mycelium by microscopic examination of tissue sections. The fungus was identified after induced sporulation in a starvation medium. The fungus grew rapidly in PCA medium at $25^{\circ} \mathrm{C}$. The identity of the fungus was determined by Dr S K Singh of the Agharkar Research Institute, Pune and by Dr G S Prasad of the Microbial type culture collection (MTCC), Institute of Microbial Technology, Chandigarh, India. The culture produced macroconidia on slender, $85 \mu \mathrm{m}$ long phialides. The macroconidia were $25-40 \times 3.75 \mu \mathrm{m}$. The culture also produced round or oval microconidia (figure 1).

Analysis of the ITS and D1/D2 26S rDNA sequence revealed $99 \%$ identity with Fusarium solani voucher NJM 0271. By the morphology of the fungal spores and 26S rDNA sequencing (figure 2), the fungus was identified as $F$. solani. This fungus is the different from the previously reported endophytic paclitaxel-producing species of Fusarium.

\subsection{Paclitaxel production}

Paclitaxel was identified based on TLC, HPLC and spectral analyses by UV, MALDI-MS and LC-ESI-MS. Figure 3 shows the HPLC chromatogram of the crude extract. Separation of the mixture of various taxanes in the extract was done with methanol:water $(70: 30 \mathrm{v} / \mathrm{v})$ over a period of $30 \mathrm{~min}$ (figure 3A). Standards of the three most abundant taxanes - baccatin III, 10-deacetyl baccatin III and paclitaxel - were resolved by this system as shown in figure 3B. Baccatin III, 10-deacetyl baccatin III and paclitaxel were identified in the crude extract by HPLC by comparison of retention time to standard peaks. Figure 4 shows the TLC analysis of the crude fungal extract. One of the fungal compounds exhibited the same $\mathrm{Rf}$ values as authentic paclitaxel, yielding a characteristic blue spot with vanillin-sulphuric acid reagent.

Figure 5 shows the UV spectrum of the HPLC fractions of the paclitaxel. The isolated compound had a UV spectrum similar to authentic paclitaxel, with a maximum absorbance at $227 \mathrm{~nm}$. MALDI-MS was used as an independent method 
for identification of paclitaxel. In the TLC purified sample, the molecular ions $\left(\mathrm{m} / \mathrm{Z}=854\right.$ of $\left.\mathrm{M}+\mathrm{H}^{+}\right)$and $(\mathrm{m} / \mathrm{Z}=876$ of $\mathrm{M}+\mathrm{Na}^{2+}$ ) corresponding to paclitaxel were present as shown in figure 6. A full-scan LC-ESI-MS chromatogram for a crude extract is shown in figure 7 . The presence of molecular ion $(\mathrm{m} / \mathrm{Z}=854)$ at the appropriate retention time confirmed that the compound was paclitaxel. The concentration of paclitaxel analysed by HPLC was $1.6 \mu \mathrm{g} / \mathrm{l}$.

\subsection{Apoptotic activity of fungal paclitaxel}

We determined whether the paclitaxel purified from $F$. solani exhibits apoptotic activity. Jurkat cells were cultured with the fungal paclitaxel for $24 \mathrm{~h}$ followed by fixing the cells, staining with ethidium bromide and estimating the apoptotic cells by FACS analyses. As seen from figure 8 , the fungal paclitaxel induced apoptosis in $66 \%$ of cells as compared with standard paclitaxel, which exhibited apoptotic activity in $51 \%$ of cells.

\section{Conclusion}

The F. solani described here is different from previously reported endophytic paclitaxel-producing fungi. Besides paclitaxel, the fungus also yielded taxanes, baccatin III and 10-deacetyl baccatin III, which are important intermediates for paclitaxel and semisynthesis of paclitaxel in industry. Knowledge of the biosynthesis of paclitaxel in fungi is necessary for determining the conditions for fermentation and metabolic engineering of the strains for overproduction of paclitaxel. We cloned a partial taxane $13 \alpha$ hydroxylase cDNA (Gen bank accession No. EF626531) from this fungus. The amount of paclitaxel produced by $F$. solani is low. Quantification by HPLC indicated that $1.6 \mu \mathrm{g}$ of paclitaxel was produced per litre of culture. The optimum culture conditions for paclitaxel production are under further study. Improved culturing techniques and the application of genetic engineering may improve paclitaxel production by $F$. solani.

\section{Acknowledgements}

Thanks are due to Mr Daniel Luscombe, Bedgebury for sending fresh stem cuttings of Taxus celebica. We are grateful to Professor Ramesh Maheshwari for useful suggestions and critical reading of the manuscript. We thank Professor Sunil K Podder for helpful discussions. We also thank Ms Akhila T S for her technical assistance. We are thankful to Professor P K Das and K R Prasad for allowing us to use their laboratory facilities. We thank Dr Omana Joy and Vamsi for the FACS analysis. This work was supported by a grant from the Department of Biotechnology, New Delhi. BVSKC is a recipient of a research fellowship from the Council of Scientific and Industrial Research, New Delhi.

\section{References}

Altschul S F, Madden T L, Schaffer A A, Zhang J, Zhang Z, Miller W and Lipman D J 1997 Gapped BLAST and PSI-BLAST: a new generation of protein database search programs; Nucleic Acids Res. 25 3389-3402

Brown D T 2003 Taxus: the genus Taxus; in Preclinical and clinical studies of the taxanes (eds) H Itokawa, H K Lee (London: Taylor and Francis) pp 387-435

Cardellina J H 1991 HPLC separation of taxol and cephalomannine; J. Liquid Chromat. 14 659-665

Croom E M 1995 Taxol: science and applications; in Taxus for taxol and taxoids (ed.) M Suffness (Boca Raton: CRC Press) pp 37-70

Fraker P J, King L E, Lill-Elghanian D and Telford W G 1994 Quantification of apoptotic events in pure and heterogeneous populations of cells using flow cytometer; in Methods in cell biology Vol 46: Cell death (eds) B Osborne and L Schwartz (New York: Academic Press) pp 57-76

Frense D 2007 Taxanes: perspectives for biotechnological production; Appl. Microbiol. Biotechnol. 73 1233-1240

Guenard D, Gueritte-Voegelein F and Potier P 1993 Taxol and taxotere: discovery, chemistry and structure-activity relationships; Acc. Chem. Res. 26160-167

Guo B H, Wang Y C, Zhou X W, Hu K, Tan F, Miao Z Q and Tang K X 2006 An endophytic taxol-producing fungus BT2 isolated from Taxus chinensis var. mairei; Afr. J. Biotechnol. $5875-877$

Li J Y, Strobel G A, Sidhu R, Hess W M and Ford E 1996 Endophytic taxol producing fungi from bald cypress Taxodium distichum; Microbiology 142 2223-2226

Murashige T and Skoog F 1962 A revised medium for rapid growth and bioassay with tobacco tissue culture; Physiol. Plant 15 473-497

Pinkerton R and Strobel G 1976 Serinol as an activator of toxin production in attenuated cultures of Helminthosporium sacchari; Proc. Natl. Acad. Sci. USA 73 4007-4011

Rogers S O and Bendich A J 1994 Extraction of total cellular DNA from plants, algae and fungi; in Plant molecular biology manual (eds) S B Gelvin and R A Schilperoort 2nd edition (Dordrecht: Kluwer Academic Publishers) pp D1-8

Stierle A, Strobel G and Stierle D 1993 Taxol and taxane production by Taxomyces andreanae, an endophytic fungus of Pacific yew; Science $260214-216$

Strobel G A, Stierle A, Stierle D and Hess W M 1993 Taxomyces andreanae a proposed new taxon for a bulbilliferous hyphomycete associated with Pacific yew; Mycotaxon 47 $71-78$

Strobel G A, Yang X, Sears J, Kramer R, Sidhu R S and Hess W M 1996 Taxol from Pestalotiopsis microspora, an endophytic fungus of Taxus wallachiana; Microbiology 142 435-440

Wang J, Li G, Lu H, Zheng Z, Huang Y and Su W 2000 Taxol from Tubercularia sp. strain TF5, an endophytic fungus of Taxus mairei; FEMS Microbiol. Lett. 193 249-253

Wani M C, Taylor H L, Wall M E, Coggen P and McPhail A T 1971 Plant antitumor agents. VI. The isolation and structure 
of taxol, a novel antileukemic and antitumor agent from Taxus brevifolia; J. Am. Chem. Soc. 93 2325-2327

Xu F, Tao W, Chang L and Guo L 2006 Strain improvement and optimization of the media of taxol-producing fungus Fusarium maire; Biochem. Eng. J. 31 67-73
Yuan J I, Jian-Nan B I, Bing Yan and Xu-Dong Zhu 2006 Taxolproducing fungi: a new approach to industrial production of taxol; Chin. J. Biotechnol. 22 1-6

Zhou D P, Ping W X and Sun J Q 2001 Study on isolation of taxol producing fungi; J. Microbiol. 21 18-20

MS received 4 October 2007; accepted 15 February 2008

ePublication: 28 March 2008

Corresponding editor: RITA MULHERKAR 